\title{
BMJ A multidisciplinary intervention to Open facilitate return to work in cancer patients: intervention protocol and design of a feasibility study
}

\author{
Iris F Groeneveld, Angela G E M de Boer, Monique H W Frings-Dresen
}

To cite: Groeneveld IF, de Boer AGEM, Frings-Dresen MHW. A multidisciplinary intervention to facilitate return to work in cancer patients: intervention protocol and design of a feasibility study. BMJ Open 2012;2:e001321.

doi:10.1136/

bmjopen-2012-001321

- Prepublication history and additional materials for this paper are available online. To view these files please visit the journal online (http://dx. doi.org/10.1136/

bmjopen-2012-001321).

Received 17 April 2012 Accepted 6 June 2012

This final article is available for use under the terms of the Creative Commons Attribution Non-Commercial 2.0 Licence; see http://bmjopen.bmj.com

\begin{abstract}
Introduction: Returning to work can be problematic for cancer survivors due to suboptimal workplace support, a heavy workload, decreased physical functioning and fatigue. The timely and permanent return to work (RtW) of cancer patients favourably influences quality of life and economic independence. Multidisciplinary interventions aimed at timely and enduring RtW are lacking. The objectives of this article are (1) to describe the protocol of an intervention aimed at RtW of cancer patients, comprising of counselling by an oncological occupational physician and supervised physical exercise in a clinical setting during treatment and (2) to present the design of the study aimed at evaluating the feasibility of this intervention.
\end{abstract}

Methods and analysis: The intervention comprises three counselling sessions with an oncological occupational physician and a 12-week moderate-tohigh intensity physical exercise programme, starting at the onset of chemotherapy. The intervention is aimed at cancer patients treated with curative intent, aged 18-60 years, employed and on sick leave. It will take place in two large medical centres in the Netherlands. The feasibility of the intervention will be evaluated as follows: the number of sessions, topics discussed and exercises executed will be registered by care providers; patients' and care providers' opinions will be assessed by questionnaires and interviews, respectively; and the proportion of invited patients that participated will be calculated.

Ethics and dissemination: The study results will be used for optimising the intervention content and may serve as a foundation for future implementation. The Medical Ethics Committees of the Academic Medical Center and the participating medical centres approved the study protocol.

Coronel Institute of Occupational Health, Academic Medical Center, University of Amsterdam, Amsterdam, The Netherlands

Correspondence to Iris Groeneveld; i.f.groeneveld@amc.nl

\section{INTRODUCTION}

In western countries, cancer prevalence has been increasing in recent decades, due primarily to early detection and improved treatment. Approximately a third of new

\section{ARTICLE SUMMARY}

\section{Article focus}

- The goals of this article are to describe the protocol of an intervention aimed at RtW of cancer patients, comprising of counselling by an oncological occupational physician and supervised physical exercise in a clinical setting during treatment and to present the design of the study used to evaluate the feasibility of this intervention.

\section{Key messages}

- Returning to work is problematic for cancer survivors due to, among other factors, suboptimal workplace support, a heavy workload, decreased physical functioning and fatigue.

- Timely and enduring RtW may favourably influence quality of life and economic independence of cancer patients.

- A multidisciplinary intervention for cancer patients consisting of counselling and physical exercise aimed at returning to work is warranted and must be evaluated for feasibility.

Strengths and limitations of this study

- The intervention is innovative in its early onset, clinical setting and the fact that both work- and physical health-related determinants of RtW are targeted.

- The feasibility of the intervention will be investigated thoroughly at the level of the patients and the care providers using qualitative and quantitative methods.

- Only one occupational health professional will be involved in the intervention, and physical exercise will not be continued after chemotherapy treatment has concluded.

cancer cases are individuals of working age. ${ }^{1}$ Most of these patients aim to return to work (RtW) eventually because RtW implies a return to 'normalcy', economic independence $^{2}$ and favourably influences quality of life. $^{3} 4$ However, RtW often appears problematic, ${ }^{5}$ and one-third of patients do not RtW at all. ${ }^{6}$ Among those who resume work, 
absence due to sickness and impairments in productivity are common. ${ }^{7}$ Work-related issues that impact RtW include a lack of practical and emotional support from the employer and care providers and a heavy workload. ${ }^{8}$ Occupational physicians (OPs) often lack information on the patient's treatment and prognosis. ${ }^{9}$ Due to limited expertise, they may be unaware of the possibilities for adjusting a patient's workplace, tasks and hours. ${ }^{10}$ As to the patients, self-reported ability to work ${ }^{6}$ and social support ${ }^{11}$ are often low. In several qualitative studies, cancer patients express a need for emotional and practical support, ${ }^{12-14}$ such as the provision of disease-related information on RtW, adjustments in workplace and working hours and development of a comprehensive plan for RtW. ${ }^{14}{ }^{15}$ Also, physical complaints, induced by the disease and its treatment, may constrain enduring RtW in cancer patients. These complaints include persistent fatigue, ${ }^{16}{ }^{17}$ reduced

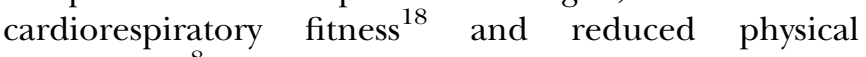
functioning. ${ }^{8}$

Although RtW is widely acknowledged as an important issue in cancer rehabilitation, studies on enhancing RtW are rare. Indeed, a recent review stated that moderatequality evidence exists for positive effects of multidisciplinary interventions involving physical exercise and education or counselling on RtW in cancer survivors. ${ }^{19}$ In the studies showing positive effects, the counsellor provided information and discussed coping skills ${ }^{20}$ or encouraged RtW. ${ }^{21}$ However, none of the studies detailed in this review focused on promoting timely or enduring RtW. ${ }^{20-22}$ Timely and enduring RtW may be facilitated by combining two strategies. The work-related barriers could be overcome by tailored counselling with an OP with expertise in cancer. Fatigue and physical functioning may be positively influenced by a physical exercise programme. ${ }^{23} 24$

Currently, it is recommended that cancer rehabilitation, including counselling and/or physical exercise, starts during treatment if the treatment is curative in intent. With regard to counselling, patients can be advised on how to deal with work while on sick leave and informed about disease- and treatment-related barriers to and possibilities for RtW. Furthermore, patients can be offered a physical exercise programme to prevent a severe decline in physical functioning and cardiorespiratory fitness. According to the American College of Sports Medicine, moderate-to-high intensity physical exercise during and after treatment is safe and can have positive effects on cardiorespiratory fitness, muscle strength and fatigue of cancer patients. The training intensity should be individualised according to pretreatment fitness, monitored throughout the exercise programme and preferably include both aerobic and resistance exercises. $^{24}$

Thus far, studies on early rehabilitation in cancer patients that combine work- and physical health-related strategies are lacking. Thus, it is important to investigate an intervention involving these two strategies. However, adding several care providers to a multidisciplinary care team implies an extensive change to usual care. Before studying the effectiveness of the intervention, a feasibility study should be performed that evaluates the execution of the intervention, patients' and care providers' opinions and the proportion of invited patients who participate. The goals of a feasibility study are to determine whether an intervention is appropriate for further testing or implementation and to identify changes necessary for optimising the intervention. ${ }^{25}$

The objectives of this article are (1) to describe the protocol of an intervention aimed at $\mathrm{RtW}$ of cancer patients, comprising counselling by an oncological occupational physician (OOP) and supervised physical exercise in a clinical setting during treatment and (2) to present the design of the study aimed at evaluating the feasibility of this intervention.

\section{METHODS AND ANALYSIS Intervention protocol}

The intervention was based on scientific literature and the opinions of care providers in the field of occupational health, oncology, sports medicine and physiotherapy. The methods and findings of the intervention development can be found in a supplementary file. Based on these findings, an intervention protocol was defined. The timing of each activity of the intervention is depicted in figure 1.

\section{Counselling by an OOP}

The counselling sessions will be performed by an OOP. The OOP has expertise on the consequences of cancer and its treatment on work and experience in counselling cancer patients on RtW. The OOP is situated at the sports medicine outpatient department at the hospital. Directly after inclusion, a sports medical assessment, to be supervised by a sports medicine physician, will be scheduled and the assessment will be followed by a counselling session with the OOP. Depending on the patient's preference, the second counselling session will be 6 or 12 weeks after the first, and the last session will be 6 weeks later or at the end of treatment. The content to be covered in the counselling sessions is presented in table 1. Most topics can be discussed in each session, and some topics will be discussed in the first session only. Additionally, four written materials will be provided: (1) a brochure on the consequences of cancer for work for the patient; (2) a comparable brochure for the employer; (3) a leaflet describing websites on cancer and work and (4) a leaflet, upon which the patient can write their attitude, self-efficacy and short- and long-term goals for RtW. Before the first counselling session, the OOP will retrieve the medical file and the results of the first sports medical assessment including peak oxygen consumption ${ }^{26}$ and information on physical disabilities from the hospital administration. In the following months, the results of the second sports medical assessment and changes in a patient's medical situation can be 


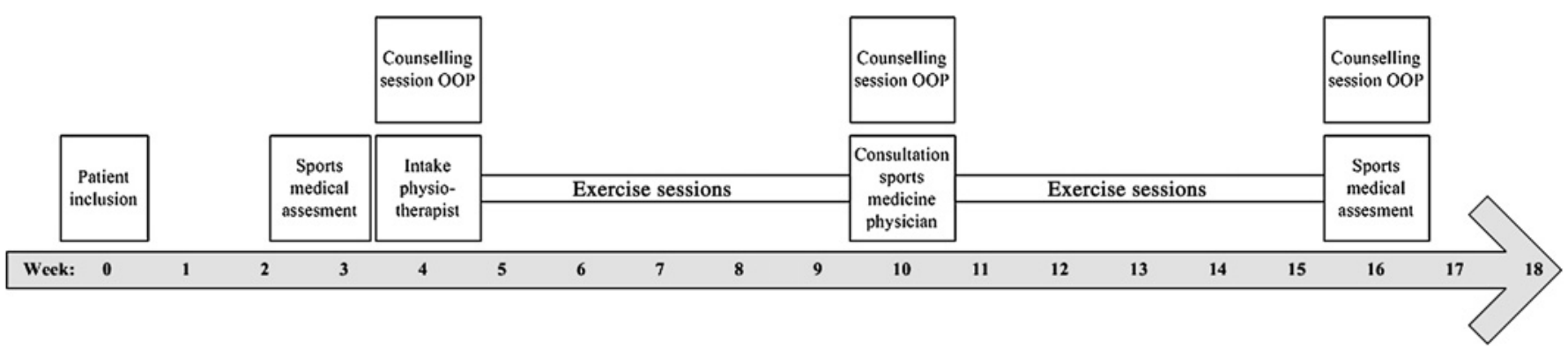

Figure 1 Timing of activities in the intervention.

retrieved. If necessary, the OOP can discuss with the oncologist, a possible referral of the patient to an additional care provider. At the end of each counselling session, the patient will receive a letter containing the notes from that session. The patient will be encouraged to hand over this letter to his company's OP to facilitate continuation of occupational support at the workplace.

\section{Supervised physical exercise}

Each physical exercise session will start and end with 8 min of interval training. The patient will pedal at a rate of $70 \mathrm{rpm}$, starting with a 1-min warm-up. For $8 \mathrm{~min}$, the intensity will alternate over $30 \mathrm{~s}$ intervals. The intensity of the interval training will be expressed as a percentage of the maximal workload $\left(\mathrm{W}_{\max }\right)$. The $\mathrm{W}_{\max }$ will be determined in the first session using an adapted version of the steep-ramp test ${ }^{27}$ and again after 4 and 8 weeks. Throughout the 12-week physical exercise programme, the intensity of interval training will increase, starting at alternations of $30 \mathrm{~s}$ at $35 \%$ and $30 \mathrm{~s}$ at $65 \%$ of $\mathrm{W}_{\max }$ in the first session to alternations of $30 \mathrm{~s}$ at $40 \%$ and $30 \mathrm{~s}$ at $70 \%$ of $\mathrm{W}_{\max }$ in the last session. Seven different exercises will be conducted during resistance training: vertical traction, leg press, bench or chest press, deltoid pulley, abdominal crunch, squats and step ups. The weights to be used will be expressed as a percentage of the 1repetition maximum (1RM). The $1 \mathrm{RM}$ will be derived from 4 to $6 \mathrm{RM}$ tests $^{28}$ performed at baseline and again after 4 and 8 weeks. Throughout the 12-week programme, the intensity of resistance exercises will increase from $65 \%$ to $75 \%$ of the $1 \mathrm{RM}$, and the number of repetitions will decrease from $3 \times 12$ to $2 \times 8$. No 1 RM value can be determined for the abdominal crunch, squats and step-up exercises. The amount of weight, and height if applicable, will be based on the patients' ability to conduct the exercise while moving continuously at a semi-fast pace. In the case of pain or discomfort, the intensity of the interval and/or resistance exercises will be adjusted. In the case of fever or vomiting, training will be discouraged. To prevent cytostatic cross-contamination, preventive measures will be taken according to the hospital's hygiene protocol. The Patient Specific Complaint list will be used to stimulate adherence to the exercise sessions. The patient will indicate one to three activities related to daily living, work or sports that he would like to improve upon.

Table 1 Counselling session numbers and topics to be discussed during counselling with the oncological occupational physician

\begin{tabular}{|c|c|c|}
\hline Topic & Session number & Content \\
\hline A & 1 & Discuss diagnosis, treatment and prognosis \\
\hline B & 1 & Discuss job type, company and current work status \\
\hline $\mathrm{C}$ & 1 & Explain Dutch laws on sickness absence and social security \\
\hline D & 1 & Advise the patient to keep in touch with colleagues and employer \\
\hline $\mathrm{E}$ & $1,2,3$ & Discuss the patient's overall physical and mental health \\
\hline $\mathrm{F}$ & $1,2,3$ & $\begin{array}{l}\text { Discuss the patient's opinions on his perceived physical and mental work ability and } \\
\text { his perceived possibilities to and barriers for RtW }\end{array}$ \\
\hline G & $1,2,3$ & $\begin{array}{l}\text { Discuss the patient's attitude towards RtW and self-efficacy regarding RtW and means } \\
\text { to improve these determinants. Assist in setting short- and long-term goals }\end{array}$ \\
\hline $\mathrm{H}$ & $1,2,(3)$ & $\begin{array}{l}\text { Check the results of the sports medical assessment to support estimation of the patient's } \\
\text { physical work ability }\end{array}$ \\
\hline I & $1,2,3$ & $\begin{array}{l}\text { Using topics } A, B, E, F, G \text { and } H \text { estimate a patient's physical and mental work ability } \\
\text { and possibilities for and barriers to RtW }\end{array}$ \\
\hline $\mathrm{J}$ & $(1), 2,3$ & $\begin{array}{l}\text { Provide advice on gradual resumption of work and relevant necessary adjustments in } \\
\text { working hours, tasks and responsibilities and recommend discussing this advice with } \\
\text { the supervisor and the company's OP }\end{array}$ \\
\hline$\underline{\mathrm{K}}$ & $(1), 2,3$ & If a return to the former job is impossible: advise the patient on reintegration to another job \\
\hline
\end{tabular}


He will score his ability to execute these activities and revise his scores after 4,8 and 12 weeks. Six weeks after the first exercise session, the patient will complete a 20 min consultation with the sports medicine physician to discuss his progress and barriers to training. During the last session, the physiotherapist will encourage the patient to remain physically active. A sports medical assessment will be performed before and after the physical exercise programme.

\section{Feasibility study}

Study design

In this feasibility study, a maximum of 130 patients will be recruited over a 2-year period. The intervention will take place in two large medical centres in the east and the west of the Netherlands. Data will be collected and analysed using both quantitative and qualitative methods during and immediately after the intervention.

\section{Study population and recruitment}

The criteria for inclusion in the feasibility study are as follows: diagnosis of cancer, undergoing adjuvant chemotherapy with curative intent, aged $18-60$ years, having paid employment, being absent from work or intending to report sick before the start of treatment. Exclusion criteria are as follows: having testicular cancer, ${ }^{11}$ unable to speak, read or write the Dutch language, having severe mental disability or being physically unable to perform exercise training. For recruitment, shortly before the onset of chemotherapy, the oncologist will provide an information letter to the patient during their first meeting. One to three weeks later, the oncology nurse will ask the patient whether he wishes to participate. If the patient agrees to participate, he will sign the informed consent form.

\section{Study outcomes and measurements}

Three aspects of the intervention will be evaluated in this feasibility study. First, the number of intervention sessions, counselling topics and exercises that are executed will be registered. Second, the patients' and care providers' opinions on the intervention will be assessed. Third, the proportion of invited patients that participate in the study will be calculated. Data on sex, date of birth, diagnosis and type of chemotherapy will be obtained from the hospital's medical records.

\section{Execution of intervention}

The number of counselling sessions performed and the topics discussed will be obtained from the letters written by the OOP after each counselling session. Similarly, the number of exercise sessions and the exercises performed will be derived from the physiotherapists' registration forms used during the physical exercise programme. The reasons for not conducting certain sessions, counselling topics or exercises will be addressed in these letters and forms by the OOP and physiotherapist, respectively. By doing so, we may reveal where the patient is noncompliant to the protocol and where the care provider fails to adhere to the protocol.
Patients' opinions on the intervention

The patients' opinions, for example, on the content and timing of the intervention and its perceived usefulness, will be assessed using two questionnaires. One will be sent within 2 weeks of completion of the first counselling session with the OOP, and the other will be sent within 2 weeks of the final sports medical assessment. The contents of these questionnaires are outlined in table 2.

\section{Care providers' opinions on the intervention}

The physiotherapists' opinions on the intervention will be assessed following completion of the intervention using 60 min focus group interviews in both participating centres separately. The interviews will cover the timing, frequency, duration, content and facilitators of and barriers to the intervention. Similarly, during a 45 min personal interview, the OOP's opinions on the same topics will be assessed. The OOP will also be asked to indicate the usefulness of the sports medical information. The personal and focus group interviews will be audio-taped and transcribed verbatim.

\section{Proportion participating}

The number of patients invited to take part in the study, obtained from the hospital medical files, will be used to calculate the proportion of invited patients who actually participate in the study. To enable the comparison of characteristics between participants and non-participants, sex, date of birth and diagnosis of all invited patients will also be retrieved from the hospital medical files.

\section{Analyses}

The means (and SDs) will be calculated for the number of counselling and exercise sessions executed; the number of topics discussed per counselling session; the number of exercises performed per exercise session and the number of written materials provided by the OOP. Additionally, the percentage of the participants who attended all three counselling sessions and all 24 exercise sessions will be calculated. To analyse patients' answers to the closed-ended questions in the questionnaires, frequencies will be calculated. In the qualitative analysis of open-ended questions, two raters will define codes independently, that is, keywords or short descriptors that describe the various topics identified. They will then discuss their findings until they reach agreement on a set of codes that covers all topics. The various codes will be presented along with the frequencies in which these codes were identified in the patients' answers. The qualitative analysis of the personal and focus group interviews with care providers will begin with open coding, that is,, assigning keywords or short descriptors to all statements, followed with axial coding, that is, structuring codes and defining transcending codes and concluded with selective coding, that is, clustering axial codes into broader categories. ${ }^{29}$ To analyse the proportion of participating patients, the number of participants will be divided by the number of patients who were invited to take part in the study. The qualitative analyses 
Table 2 The content of two evaluation questionnaires aimed at determining the patient's opinion on the intervention

\begin{tabular}{|c|c|}
\hline Outcome variable & Measurement \\
\hline \multicolumn{2}{|l|}{ Questionnaire 1: after the first counselling and exercise session } \\
\hline $\begin{array}{l}\text { Opinion on the timing of the sports medical assessment, intake } \\
\text { to the study and first counselling and exercise session }\end{array}$ & Too early-too late (3-point scale) \\
\hline Opinion on the content of the first counselling and exercise session & $\begin{array}{l}\text { Very pleasant-very unpleasant } \\
\text { (4-point scale) }\end{array}$ \\
\hline \multicolumn{2}{|l|}{ Questionnaire 2: upon completion of the intervention } \\
\hline Opinion on the content of the counselling and exercise sessions & $\begin{array}{l}\text { Very pleasant-very unpleasant } \\
\text { (4-point scale) }\end{array}$ \\
\hline Opinion on the duration of the intervention as a whole & Too short-too long (3-point scale) \\
\hline Opinion on the overall competence of the OOP and physiotherapist & Report mark (10-point scale) \\
\hline $\begin{array}{l}\text { Perceived competence of the OOP with regard to various counselling } \\
\text { strategies, for example, listening well, providing useful information, } \\
\text { adequately answering questions }\end{array}$ & $\begin{array}{l}\text { Highly disagree-highly agree } \\
\text { (5-point scale) }\end{array}$ \\
\hline $\begin{array}{l}\text { Perceived usefulness of counselling components, for example, } \\
\text { discussing possibilities for and barriers to RtW }\end{array}$ & $\begin{array}{l}\text { Very useful-not useful } \\
\text { (3-point scale)/not applicable }\end{array}$ \\
\hline Most and least useful components of the counselling and exercise sessions & Open question \\
\hline $\begin{array}{l}\text { Perceived interference between medical treatment and counselling } \\
\text { and/or exercise sessions }\end{array}$ & Yes/no \\
\hline $\begin{array}{l}\text { Main reasons for not attending or not finishing counselling and } \\
\text { exercise sessions }\end{array}$ & Open question \\
\hline Fulfilment of expectations regarding intervention as a whole & Yes-partly-no \\
\hline Causes of unfulfilled expectations regarding intervention as a whole & Open question \\
\hline Suggestions for improvements of the counselling and exercise sessions & Open question \\
\hline
\end{tabular}

of the personal and focus group interviews will be conducted using MAXQDA (V.2007, Marburg, Germany).

\section{ETHICS AND DISSEMINATION}

We aim to disseminate the results of this study by publication in peer-reviewed scientific journals and presentations at international conferences. The Medical Ethics Committee of the Academic Medical Center and the Medical Ethics Committees of the participating medical centres approved the study protocol. All patients will provide signed informed consent prior to inclusion in the study.

\section{DISCUSSION}

The goals of this article were to describe the protocol of a multidisciplinary intervention for cancer patients aimed at RtW and to present the design of a study used to investigate the feasibility of the intervention.

The intervention was based on scientific literature and the opinions of care providers in the field of occupational health, oncology, sports medicine and physiotherapy. With regard to the first aim, the intervention will consist of three counselling sessions with an OOP and a 12-week, twice-weekly, moderate-to-high intensitysupervised physical exercise programme, both of which are conducted in the clinical setting. With regard to the second aim, a feasibility study will be conducted involving a maximum of 130 cancer patients who are treated with curative intent, aged 18-60 years, employed and on sick leave. To determine the feasibility of the intervention, three aspects will be evaluated: the extent to which the intervention is executed, the patients' and care providers' opinions on the intervention and the proportion of invited patients who participate in the study. Data will be collected and analysed using quantitative and qualitative methods.

In the majority of previous studies on RtW in cancer survivors, encouragement, education, counselling or advice alone had no significant effects. ${ }^{30}$ In contrast, moderate-quality evidence existed for the effectiveness of education or counselling combined with physical exercise, ${ }^{19}$ although exercise in those studies was of lower intensity $^{20}$ than in our feasibility study, or homebased only. ${ }^{21}$ Recently, physical exercise of high intensity showed a statistically significant positive effect on the number of working hours, ${ }^{31}$ presumably by influencing fatigue and physical functioning. Thus, we expect that combining counselling with moderate-to-high intensity physical exercise will be an appropriate strategy for enhancing RtW.

Several strengths of the intervention can be mentioned. First, the timing of the intervention is advantageous. At an early stage, the advice of an OOP on dealing with work while on sick leave, on recruitment of social support from the workplace and making adjustments in working hours and tasks may increase the likelihood of a timely and enduring RtW. ${ }^{8}$ Additionally, an early start in physical exercise may contribute to maintenance of physical functioning and prevention of fatigue. $^{32}$ Thereby, the capacity to perform activities of 
daily living during treatment can be enhanced and a return to pre-illness conditions following treatment can be facilitated. Moreover, physical exercise may reduce disease symptoms, ${ }^{33}$ anxiety and self-esteem ${ }^{34}$ during treatment. Second, the setting of the intervention is favourable. Offering both treatment and rehabilitation efforts within the hospital is expected to be convenient for the patient and may enhance his or her compliance. Furthermore, the OOP can obtain (sports-) medical information easily from the hospital administration. The study design is also advantageous. In contrast to most other studies, ${ }^{33}$ patients with a broad range of cancer diagnoses will be recruited, optimising the generalisability of the results. Furthermore, recruitment via oncologist may enhance the proportion of patients who participate because trust in one's medical doctor may be a cause for participating. ${ }^{35}$ Both quantitative and qualitative methods will be applied in data collection and analysis to obtain a thorough picture of the feasibility of the intervention. ${ }^{25}$ A limitation of the intervention is that only one OOP will be used for the occupational part of the intervention. Currently, this OOP is one of the few OOPs skilled in occupational rehabilitation of cancer patients in the Netherlands. In future interventions, involvement of more OOPs is recommended. We would also recommend continuation of physical exercise following treatment to further maintain and increase cardiorespiratory fitness.

In this article, we described the protocol of a multidisciplinary intervention aimed at RtW, and we also presented the study design to investigate its feasibility. The results of the feasibility study may be used for optimisation of the intervention and may serve as a basis for implementation of the intervention.

\begin{abstract}
Acknowledgements We are grateful to the Alpe d'Huzes Foundation/Dutch Cancer Society for funding the study. This study is part of the A-CaRe 2 Move program: http://www.a-care.org/. The authors thank the A-CaRe 2 Move Research Collaboration. Furthermore, Mr Th. C. de Winter, MD, MSc; Mr T. Brandon, MD, MSc; Mr G. Schep, MD, PhD; Mr T. Rejda, MD, MSc, and the physiotherapists and sports medicine physicians of the Medisch Centrum Haaglanden and Isala Klinieken are acknowledged for their contribution during the design of the intervention.
\end{abstract}

Contributors IFG wrote the manuscript. AGEMdB and MHWF-D were involved in critically revising the manuscript and providing intellectual input. All authors contributed to the development of the intervention and the study design. All authors read and approved the final manuscript.

Funding This work was supported by the Alpe d'Huzes Foundation/Dutch Cancer Society, grant number NKI 2010-4854.

Competing interests None.

Ethics approval Ethics approval was provided by Medical Ethics Committee Amsterdam Medical Center.

Provenance and peer review Not commissioned; externally peer reviewed.

\section{REFERENCES}

1. World Health Organization: International Agency for Research on Cancer. GLOBOCAN: Cancer Incidence and Mortality Worldwide in 2008. 2010. http://globocan.iarc.fr (accessed 18 Oct 2011).

2. Butler RJ, Johnson WG, Gubler T. Economic burden. In: Feuerstein $\mathrm{M}$, ed. Work and Cancer Survivors. 1st edn. New York: Springer, 2009:25-72.
3. Bieri S, Roosnek E, Helg C, et al. Quality of life and social integration after allogeneic hematopoietic SCT. Bone Marrow Transplant 2008;42:819-27.

4. Ferrell B, Grant M, Schmidt GM, et al. The meaning of quality of life for bone marrow transplant survivors. Part 2. Improving quality of life for bone marrow transplant survivors. Cancer Nurs 1992;15:247-53.

5. Roelen CA, Koopmans PC, Schellart AJ, et al. Resuming work after cancer: a prospective study of occupational register data. J Occup Rehabil 2011:21:431-40.

6. de Boer AG, Verbeek JH, Spelten ER, et al. Work ability and returnto-work in cancer patients. Br J Cancer 2008;98:1342-7.

7. Goetzel RZ, Long SR, Ozminkowski RJ, et al. Health, absence, disability, and presenteeism cost estimates of certain physical and mental health conditions affecting U.S. employers. J Occup Environ Med 2004;46:398-412.

8. Taskila T, Lindbohm ML. Factors affecting cancer survivors' employment and work ability. Acta Oncol 2007;46:446-51.

9. Verbeek J, Spelten E, Kammeijer M, et al. Return to work of cancer survivors: a prospective cohort study into the quality of rehabilitation by occupational physicians. Occup Environ Med 2003;60:352-7.

10. Amir Z, Brocky J. Cancer survivorship and employment: epidemiology. Occup Med (Lond) 2009;59:373-7.

11. Spelten ER, Sprangers MA, Verbeek JH. Factors reported to influence the return to work of cancer survivors: a literature review. Psychooncology 2002;11:124-31.

12. Tamminga SJ, de Boer AG, Verbeek JH, et al. Breast cancer survivors' views of factors that influence the return-to-work process-a qualitative study. Scand J Work Environ Health 2012;38:144-54.

13. Taskila T, Lindbohm ML, Martikainen R, et al. Cancer survivors' received and needed social support from their work place and the occupational health services. Support Care Cancer 2006;14:427-35.

14. Tiedtke C, de Rijk A, Dierckx de Casterlé B, et al. Experiences and concerns about 'returning to work' for women breast cancer survivors: a literature review. Psychooncology 2010;19:677-83.

15. Yarker J, Munir F, Bains $\mathrm{M}$, et al. The role of communication and support in return to work following cancer-related absence. Psychooncology 2010;19:1078-85.

16. Sesto ME, Simmonds MJ. Fatigue, pain and physical function. In: Feuerstein M, ed. Work and Cancer Survivors. 1st edn. New York: Springer, 2009:123-46.

17. Weis J. Cancer-related fatigue: prevalence, assessment and treatment strategies. Expert Rev Pharmacoecon Outcomes Res 2011;11:441-6.

18. Kaleta D, Makowiec-Dabrowska T, Jegier A. Leisure-time physical activity, cardiorespiratory fitness and work ability: a study in randomly selected residents of Lodz. Int J Occup Med Environ Health 2004; 17:457-64.

19. de Boer AG, Taskila T, Tamminga SJ, et al. Interventions to enhance return-to-work for cancer patients. Cochrane Database Syst Rev 2011;(2):CD007569.

20. Berglund G, Bolund C, Gustafsson UL, et al. One-year follow-up of the 'Starting Again' group rehabilitation programme for cancer patients. Eur J Cancer 1994;30A:1744-51.

21. Maguire $P$, Brooke $M$, Tait $A$, et al. The effect of counselling on physical disability and social recovery after mastectomy. Clin Oncol 1983;9:319-24.

22. Burgio KL, Goode PS, Urban DA, et al. Preoperative biofeedback assisted behavioral training to decrease post-prostatectomy incontinence: a randomized, controlled study. J Urol 2006;175:196-201

23. McNeely ML, Campbell KL, Rowe $\mathrm{BH}$, et al. Effects of exercise on breast cancer patients and survivors: a systematic review and metaanalysis. CMAJ 2006;175:34-41.

24. Schmitz KH, Courneya KS, Matthews C, et al. American College of Sports Medicine roundtable on exercise guidelines for cancer survivors. Med Sci Sports Exerc 2010;42:1409-26.

25. Bowen DJ, Kreuter M, Spring B, et al. How we design feasibility studies. Am J Prev Med 2009;36:452-7.

26. European Respiratory Society (ERS) Task Force on Standardization of Clinical Exercise Testing. Clinical exercise testing with reference to lung diseases: indications, standardization and interpretation strategies. Eur Respir J 1997;10:2662-89.

27. De Backer IC, Schep G, Hoogeveen A, et al. Exercise testing and training in a cancer rehabilitation program: the advantage of the steep ramp test. Arch Phys Med Rehabil 2007;88:610-16.

28. Dohoney $P$, Chromiak JA, Lemire $D$, et al. Prediction of one repetition maximum (1RM) strength from a 4-6 RM and a 7-10 RM submaximal strength test in healthy young adult males. J Exerc Physiol 2002;5:54-9.

29. Boeije $\mathrm{H}$. Methods and techniques in qualitative analysis. In: Analysing in Qualitative Research. 1st edn. Den Haag: Boom Onderwijs, 2005:84-117. 
30. Tamminga SJ, de Boer AG, Verbeek JH, et al. Return-to-work interventions integrated into cancer care: a systematic review. Occup Environ Med 2010;67:639-48.

31. Thijs KM, de Boer AG, Vreugdenhil G, et al. Rehabilitation using highintensity physical training and long-term return-to-work in cancer survivors. J Occup Rehabil 2012:22:220-9.

32. American College of Sports Medicine. ACSM's Guidelines for Exercise Testing and Prescription. 8th edn. Philadelphia: Lippincott Williams \& Wilkins, 2009.
33. Schmitz KH, Holtzman J, Courneya KS, et al. Controlled physical activity trials in cancer survivors: a systematic review and metaanalysis. Cancer Epidemiol Biomarkers Prev 2005;14:1588-95.

34. Speck RM, Courneya KS, Masse LC, et al. An update of controlled physical activity trials in cancer survivors: a systematic review and meta-analysis. J Cancer Surviv 2010;4:87-100.

35. Ellis PM. Attitudes towards and participation in randomised clinical trials in oncology: a review of the literature. Ann Oncol 2000;11:939-45. 